\title{
GAIA Prenatally Diagnosed Congenital Microcephaly Level of Diagnostic Certainty
}

National Cancer Institute

\section{Source}

National Cancer Institute. GAIA Prenatally Diagnosed Congenital Microcephaly Level of

Diagnostic Certainty. NCI Thesaurus. Code C128695.

A classification of maternal and fetal outcomes relating to diagnosing congenital microcephaly prenatally, developed by the Global Alignment of Immunization safety Assessment in pregnancy, based on the extent to which the diagnosis has been confirmed. 